\section{Hot topics in venous ulcer treatment: an international survey}

\author{
Stefano Ricci, ${ }^{1}$ Francesco Maria Serino, ${ }^{1}$ \\ Moro Leo, ${ }^{1}$ Fausto Passariello ${ }^{2}$ \\ 'Department of Geriatrics, University \\ “Campus Bio-Medico", Roma; ${ }^{2}$ Centro \\ Diagnostico Aquarius, Napoli, Italy
}

\section{Abstract}

Compression is the most effective treatment to promote skin ulcer healing, although there are as many different methods of performing a leg compression as the number of phlebologists, each one having personal tricks, solutions, habits. Conversely, though dressings may be done in different ways, none is considered the standard solution. We asked few (18) questions to physicians involved in this field, in Italy and abroad, looking for some common feature. The analysis of 100 replies indicated the average treatment: compression made by multilayer bandage when edema is present, knee long stocking when edema is removed, mostly based on patient's agreement. Escharectomy should be done when ulcer bed is covered by necrotic tissue. Advanced wound care is used, with dressing changed several times a week. For highly exuding ulcers, a specific dressing is used or frequent changing is performed. The treatment in early stage of disease is done by the physician while subsequently the dressings may be performed by the patient himself, nurses or doctors. Usually no medical treatments are associated. The cost of a single dressing is often lower than 10 Euro, without any reimbursement. The compression devices aren't reimbursed as well. The expected compression pressure is $30 / 40 \mathrm{~mm} \mathrm{Hg}$. Daytime compression is not reduced at night-time. The patient is always invited to walk. The same compression is used if the ABPI is $<1$. However the survey shows that several variations in the cookbook may occur, sometimes even contradictory.

\section{Introduction}

There are many patients with venous ulcer and, at the same time, a relatively high number of practitioners taking care of these patients. Many cases are treated by NHS services, but a great number are very well managed in private practice setting. For such a socially relevant problem, the Italian Heath Care System does not provide reimbursement for compression hosiery and dressings easily leading to an under treatment of this disease. Furthermore, this topic is particularly neglected in schools of medicine, being committed to teachers' good willing.

These are some of the reasons for the extreme variability of venous ulcer treatments in our country. Although compression is considered the most effective therapy to achieve ulcer healing all over the world, there are probably as many different methods of performing a leg compression as the number of phlebologists, each one having personal tricks, solutions, habits. Conversely, although dressings may be done according to different modalities, none is considered the standard solution.

Guidelines and Consensus Papers concerning compression for ulcers treatment suggest generic principles but confirm the lack of a common management of the different aspects of treatment (level and methods of compression, ulcer dressing, medical treatment, frequency of dressing, etc.).

Trying to find some common features of ulcer treatment we asked few (18) short questions to some physicians involved in this field, in Italy and abroad, with particular attention to costs and reimbursements.

\section{Materials and Methods}

A questionnaire was submitted by Vasculab mailing list in occasion of the International Vasculab Event Compression 2012, Sorrento, Italy and successively sent by email to 500 doctors involved in the field. Ninety-nine replies have been considered as valid: 63 from Italy, 9 from USA, 3 from United Kingdom, 2 from Brazil, France, Germany, Hungary Venezuela, 1 from Argentina, Australia, Belgium, Chile, Colombia, El Salvador, India, Moldova, Poland, Romania, Spain, Sweden, Switzerland, Turkey (Appendix). While the number of Italian respondents may be considered sufficient to represent a sample of Italian caregivers, the number of international participants is obviously insufficient to be representative of their countries, but may still be useful for a general comparison of the different habits in the cookbook.

\section{Questions and answers}

Question 1: How is the compression performed when the leg affected by an ulcer has an edema?

a. By a LONG stretch bandage (elastic bandage)

b. By a SHORT stretch bandage (ideal type bandage)
Correspondence: Stefano Ricci, Corso Trieste 123, 00198 Roma, Italy.

Tel. +39.06.8551523.

E-mail: varicci@tiscali.it

Key words: compression, venous ulcers, wound healing, treatments survey.

Contributions: SR, concept and design, manuscript final approval; FS, data acquisition and analysis, manuscript revision; LM, manuscript revision; FP, data acquisition, manuscript revision.

Conflict of interests: the authors declare no potential conflict of interests.

Conference presentation: the results of this survey were shown at the International Vasculab Event "Compression 2012", held in Sorrento, Italy in May 11th, 2012. "Vasculab" is a Phlebology Forum (www.vasculab.it) organized by one of the Authors (FP).

Received for publication: 31 October 2012.

Revision received: 19 December 2012.

Accepted for publication: 7 January 2013.

This work is licensed under a Creative Commons Attribution 3.0 License (by-nc 3.0).

(C)Copyright S. Ricci et al., 2012

Licensee PAGEPress, Italy

Veins and Lymphatics 2012; 2:e10

doi:10.4081/vl.2012.e10

c. By a MULTI LAYERS bandage

d. By an ADHESIVE bandage

e. By a KNEE LENGHT STOCKING $(\mathrm{K} 1, \mathrm{~K} 2, \mathrm{~K} 3)$

f. Other

A shown in Figure 1, 52\% of responders perform a multilayer bandage; $20 \%$ prefers a short stretch bandage; $4 \%$ use adhesive bandages, $3 \%$ Unna boot and $1 \%$ a cohesive bandage. $12 \%$ of physician prefers elastic compression (stockings 4\%, long stretch bandages (8\%).

Short stretch compression is the most employed compression when an edema is present for its high working pressure. ${ }^{1,2}$ Multilayer bandage is widely appreciated for its adaptability to any shape, well fitting to the progressive volume reduction of the leg and highly effective. ${ }^{1}$ These systems generally combine padding and elastic or inelastic materials and are widely accepted as being effective in achieving strong compression. Also a short stretch bandage may be very effective (high working pressure), with the advantage of being washable and reusable but with disadvantage of loosing quickly its strength as the edema reduces, thus requiring more frequent repositioning, in order to prevent bandage slippage. $^{2}$ The adhesive bandage loose pressure as well but do not slip, even if they cannot be reutilized. Three responder prefer Unna boot 
that has similar behavior. ${ }^{2}$ The long stretch bandage, although elastic (i.e. able to adapt to the volume reduction) is less effective for its reduced stiffness and for possible skin damage if applied with strong pressure, ${ }^{2}$ knee stockings are probably preferred as easy to use, but they cannot adapt to the circumferential reduction of the limb unless using progressive smaller sizes, with disproportioned costs.

\section{Question 2: How is the compression performed when edema is reduced?}

a. By a LONG stretch bandage (elastic bandage)

b. By a SHORT stretch bandage (ideal type bandage)

c. By a MULTI LAYERS bandage

d. By an ADHESIVE bandage

e. By a KNEE LONG STOCKING (K1, K2, K3)

f. Other (specify inside the final open space)

After edema resolution, the preference is in part transferred from inelastic compression (still used by about $40 \%-b+c+b+$ some of f) to knee stocking (49\%) or other elastic tools (2\% $\mathrm{a}+2 \% \mathrm{f}$ ) (Figure 2).

\section{Question 3: If you replied "e", please specify the rationale of your choice...}

a. Ease for putting on

b. Efficacy

c. Costs

d. Comfort

e. Patient's agreement

f. Absence of exudates

g. Absence of edema

h. Other (specify inside the final open space)

For responders favoring of a compression performed by a knee stocking (Figure 3), the choice is mostly due to patients compliance (40\%) and comfort (6\%) or, secondarily (15\%), because easier to put on (thus considered more comfortable), and only $15 \%$ because more effective.

In consensus papers, hosiery is not considered the first choice even if some studies have claimed elastic stocking being as effective as inelastic bandages. ${ }^{2,3}$ Due to the lack of padding it is not considered a practical option for patients at high risk of pressure damage, with large ulcers or high amount of exudates. ${ }^{2}$ Strong compression hosiery is indicated as a first-line treatment for patients with small, uncomplicated ulcers, who wish to self care requiring daily skin care, or find bandages too hot or bulky; self application using an application device is an advantage but can be occasionally difficult. ${ }^{2}$ The reply: easier to put on (15\%) seems not considering the fact that a compression stocking needs to slip over on a painful area compared to apply a bandage. A two-layer system, chosen by two responders, may be the good idea: usually the first stocking $(10-24 \mathrm{mmHg})$ holds the dressing in place and the second one $(20-30 \mathrm{mmHg}$ ) can be removed at night, with a total pressure that may go up to $40 \mathrm{mmHg}^{2}$

Multi layer bandaging exerting strong compression is generally suggested for the treatment of venous leg ulcers. ${ }^{4}$ According to a Cochrane review in ulcer treatment the higher the pressure the higher the ulcer healing rate. ${ }^{5}$ This seems best achieved in our survey by short stretch bandages (18\%), adhesive bandages (9\%) or multi layer bandages (6\%) Elastic (long stretch) bandaging (2\%) seems the less suitable for achieving high pressures for long periods due to the risk of pressure skin damage and for the higher resting pressure when the patient is laying down. ${ }^{2}$

\section{Question 4: An escharectomy} should be done...

\section{a. Always}

b. When necrotic tissue is present

c. When an infection is present

d. When pain is present

e. Other (specify inside the final open space)

In our survey $93 \%$ of responders make a debridement, but $20 \%$ who does always a debridement shows a quite aggressive attitude (Figure 4). It would have been interesting to study the general setting (hospital versus doctor's office) of these practitioners and also their relationship with the private practice. Interestingly, some few colleagues never find necessary to perform the ulcer debridement providing that compression is sufficiently effective. One colleague suggests eliminating the bacterial biofilm by Laser 808 after coloring the wound by methylen blue.

Necrotic tissue over the wound is rare in venous ulcers, more frequent in case of ischemic pathophysiology. Initial debridement is required to remove necrotic tissue, excessive bacterial burden, and dead and senescent cells burden that can all inhibit wound healing. ${ }^{6}$ Care givers can choose among several debridement methods including sharp, enzymatic, mechanical, biological, or autolythic. More than one debridement method may be appropriate. ${ }^{6}$ Sharp debridement often is the preferred. However, the method debridement may depend on the status of the wound, the ability of the care giver, the general condition of the patient, and the professional licensing restrictions. ${ }^{6}$ Excessive debridement can result in a reactivation of the inflammatory process with a consequent influx of inflammatory cytokines. ${ }^{6}$

To our best knowledge, there are no studies, which compare debridement with no debridement in the management of venous ulcers. There is a lack of data to clarify whether mechanical, chemical or bio-surgical methods are most appropriate in this patient group. ${ }^{4}$

\section{Question 5: Which is your treatment} approach to the wound care?

a. Advanced wound care

b. Normal Saline Solution

c. Antibiotics

d. Antiseptics

e. Steroids

f. Other (specify inside the final open space)

As shown in Figure 5, 60\% of responders $(\mathrm{a}+\mathrm{f})$ use advanced wound dressings or an empirical mixture of topical agents suggested by anecdotal experience (e.g. silver ions, colloidal gels, sugar or honey, vaseline, zinc oxide, soap washings), often citing TIME principles. ${ }^{7}$ Normal saline is used by about $30 \%$ of caregivers. Local antibiotics, antiseptics and steroids are not frequently employed.

From the patients perspective the local ulcer treatment, more than bandaging, is the most important aspect of venous ulcer treatment, as they see the ulcer dressing the real source of healing. Often the same attitude may be found in practitioners, believing that some specific dressing may accelerate healing. This may explain the variety of topical agents suggested.

Guidelines and reviews for wound treatments $^{6-10}$ suggest that in any case dressing should be cost effective. Cost-effectiveness must be taken into maximal consideration; the risk could be that patients or nurses preferences push into using dressings slightly more effective but much more expensive. ${ }^{9}$ A modern dressing should maintain a moist wound environment to promote autolythic debridement, cell migration and matrix synthesis so accelerating the wound. ${ }^{6}$ Moist wound environment also reduces pain, while dry dressings are considered harmful and can cause dehydration of the wound. ${ }^{7}$

A wide variety of dressings is available, but the evidence for their effectiveness is controversial so that the use of dressings is not always recommended..$^{9,10}$

Sugar or honey, suggested by three respondents could deserve some interest, since it combines efficacy and low cost..$^{11,12}$

\section{Question 6: How frequently is the dressing changed on the average?}

a. When required

b. Every day

c. Several times a week

d. Once a week

e. Other (specify inside the final open space)

About $60 \%$ of responders change the dressing quite frequently $(a+b+c,+$ some e), usually because of exudation, pain or smell. Most of those who chose the open answer (e) seems to prefer a long change interval when possible; about $1 / 4$ of the responders prefer weekly dressing change interval. Interestingly, about $20 \%(\mathrm{~b}+\mathrm{e})$ chose a daily changing (Figure 6). 


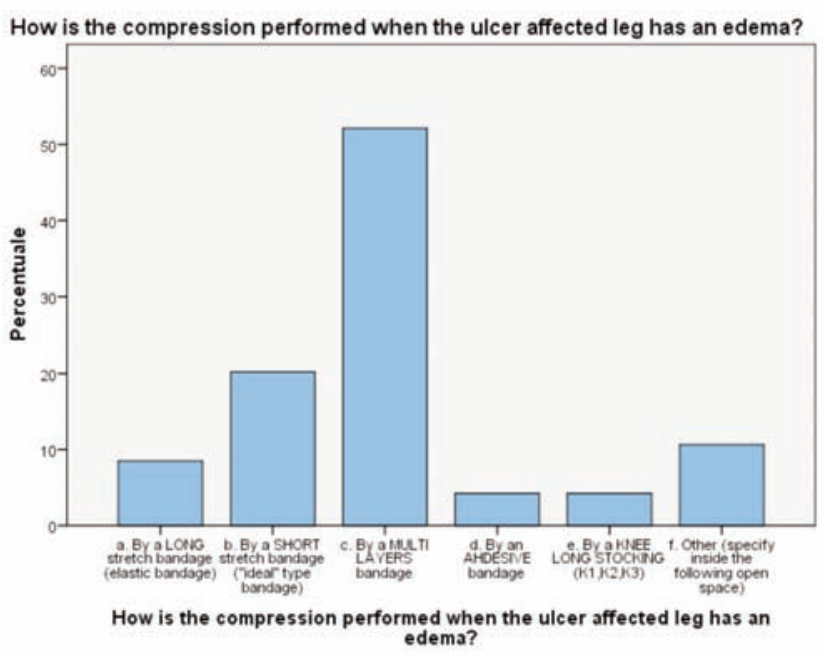

Figure 1. Methods of compression when the leg affected by an ulcer has an edema.

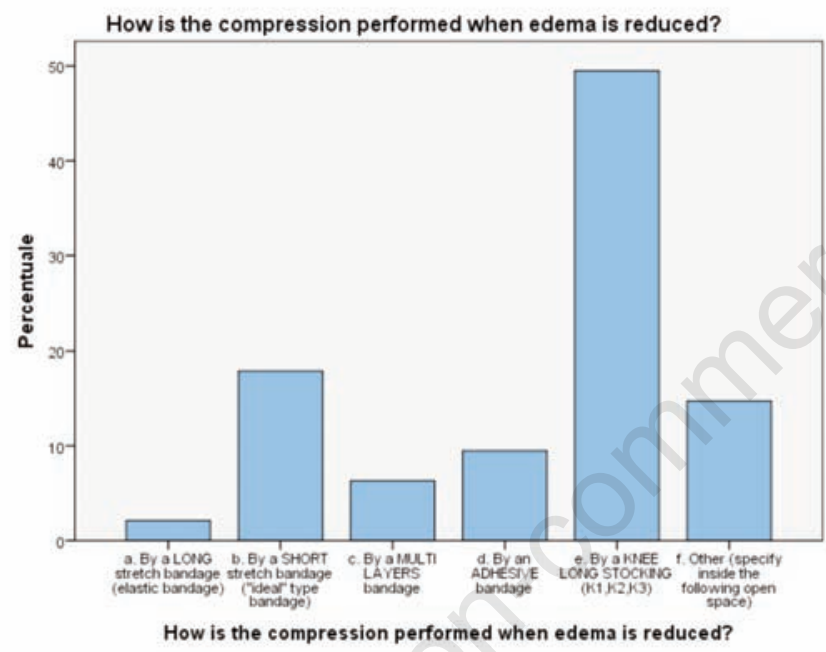

Figure 2. Methods of compression when edema is reduced.

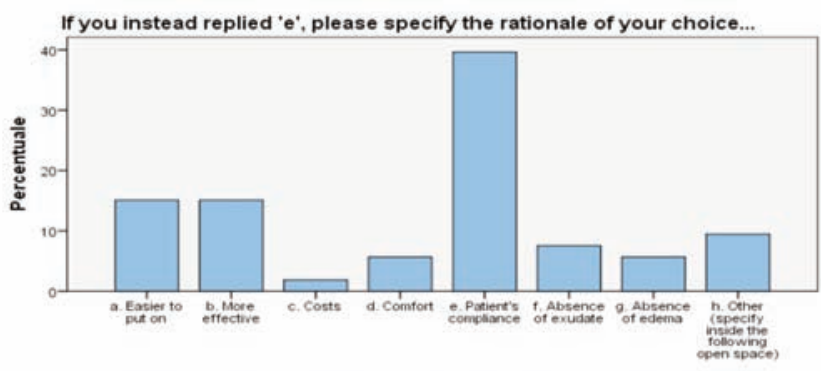

Figure 3. Response in favor of compression with knee stocking.

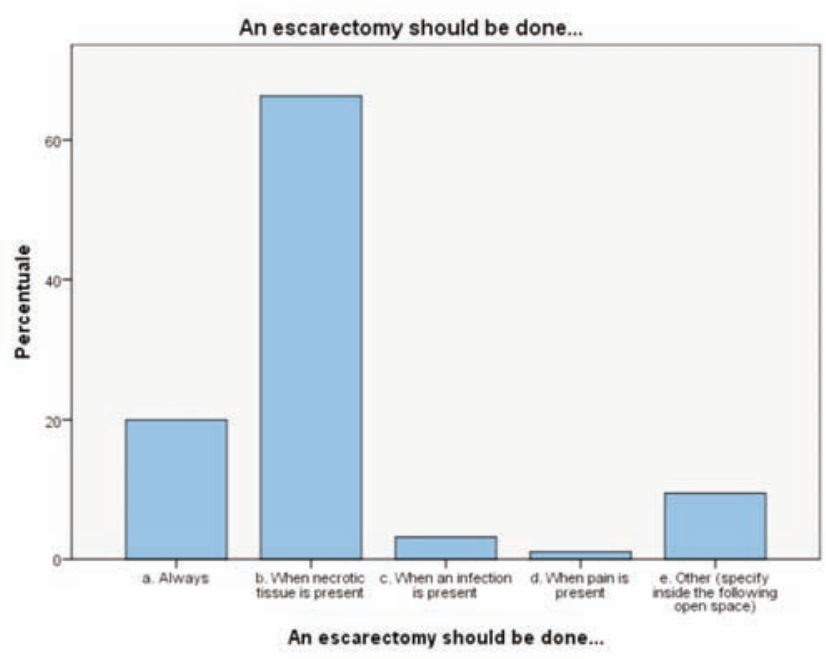

Figure 4. Cases in which an escharectomy should be performed.

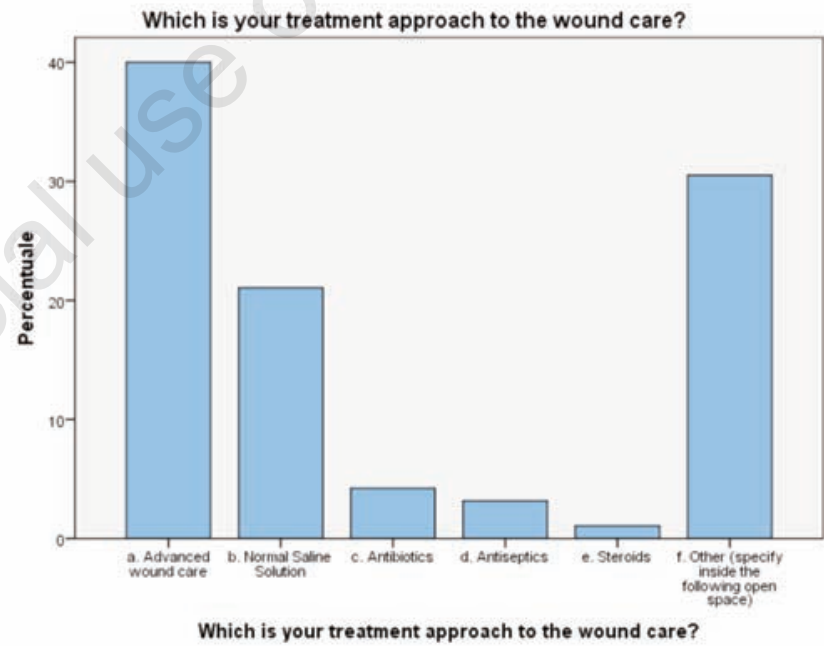

Figure 5. Treatment approaches to wound care.

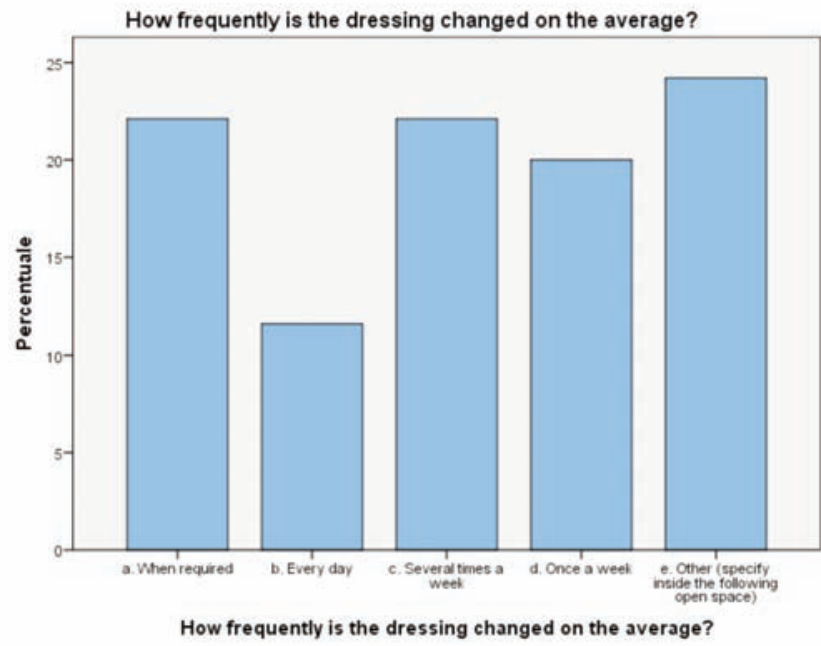

Figure 6. Average frequency of changing dressing. 
Peri-wound maceration and continuous skin contact with wound exudates can enlarge the wound and impede healing, requiring dressing change; however, when granulation tissue is achieved, the frequency of dressing change can be reduced to one per week (or even more) to allow a faster wound re-epithelization. ${ }^{6}$

In fact, the dressing changing causes a temperature fall of the wound, hampering cellular mitosis for at least $16 \mathrm{~h}$. This imply that frequent dressing change will favor debridement but long intervals between dressings will favor granulation tissue and re-epithelization. ${ }^{6}$

Furthermore, daily medication is hardly managed in an office wound healing setting, due to excessive patients travelling costs. ${ }^{5}$ On the other hand, patients can be treated at home, but with higher costs for the community or the patients itself.

\section{Question 7: When heavy exudation is present, what is the best therapeutic option? \\ a. Bed rest \\ b. Frequent changes of dressing \\ c. Specific dressing \\ d. Medical therapy \\ e. Other (specify inside the final open space)}

This question is partly related to the previous one (No. 6) since the frequent dressing change may be a method to control wound exudation $(45 \% \mathrm{~b}+\mathrm{e})$; a special dressing is proposed by $32 \%$ of responders (presumably alginate based dressings). Bed rest is the best choice for about $18 \%$ of respondents, most in association to the other methods (Figure 7).

Frequent dressing is obviously the simplest solution; however in some cases bed rest (with leg elevation if possible) may be prescribed, especially during early phases. Even if bed rest may be considered inappropriate in a patient affected by venous insufficiency (due to stasis, limited muscle pumping, slow metabolism, limited breathing, etc.), it helps to eliminate liquid from the distal limb, reducing the need of frequent dressing change and accelerating the wound healing process. ${ }^{13}$

\section{Question 8: Who performs the initial} treatment...

a. The patient (or a relative)

b. A doctor

c. A nurse

Usually the initial treatment is done by a doctor (90\%), rarely by a nurse $(6 \%)$, but curiously, also by patients (4\%), probably meaning that patients do the first attempts (Figure 8).

\section{successive treatment... \\ a. The patient (or a relative) \\ b. A doctor \\ c. A nurse}

Question 9: Who performs the
Successive treatment is done by doctors only in $40 \%$ of cases, while nurses take over doctors in $43 \%$ of cases and patients in $17 \%$ of cases (Figure 9A).

Nurses can do a great job in systems where a good nursing wound care management is organized, which is not frequent in Italy. Patient's self treatment (or by his/her relatives) is recommended only when ulcer is nearly healed, and only when the patient has shown a good compliance (Figure 9B).

A British survey reported that $71 \%$ of practitioners are the only responsible for determining the patient's venous ulcer treatment plan. ${ }^{14}$

\section{Question 10: Which medical} treatment is usually associated?

a. Antibiotics

b. NSAIDs

c. Supplements

d. LMWH

e. None

f. Other (specify inside the final open space)

As shown in Figure 10, $32 \%$ of responders avoid association of a general and a local treatment. Antibiotics are the most frequent medical association for 25\% $(a+f)$. Current prescribing guidelines recommend that antibacterial preparations should only be used in cases of clinical infection and not for bacterial colonization. ${ }^{4}$

Dietary supplements are suggested by $10 \%$ of responders, although there is still insufficient evidence to recommend them; LMWH reported by $8 \%$, is generally suggested for microangiopatic ulcers (necrotic angiodermatitis, atrophie blanche). ${ }^{15}$

\section{Question 11: What is the cost of a} single dressing (in Euro), not including the compression items

a. $<10$

b. $<20$

c. $<30$

d. $<40$

e. $\geq 50$

For most of the responders, the cost of a single dressing is under $20 €(67 \%)$ and for $41 \%$ even under $10 €$ but more than $12 \%$ of replies report costs higher than $30 €$ (Figure 11).

Saline solution and not adhesive gauze seem to be the lowest cost dressings.

\section{Question 12: Is this charge}

reimbursed or supplied by the

Sanitary System of your country at a. $100 \%$

b. $80 \%$

c. $\leq 50 \%$

d. 0

Low cost is important when we consider that venous ulcers require several dressing renew- al during three months (that is the average the time required to achieve healing in most of cases) and it's even more important if we consider that more than half of replies report no reimbursement rates, and strongly limited in other $21 \%$. It is amazing that more than $12 \%$ of replies report costs higher than $30 €$ for a single dressing (Figure 12).

\section{Question 13: Compression devices (bandages, stockings) are reimbursed by the NHS of your country? \\ a. Yes \\ b. Yes but... (specify inside the final open space) \\ c. No}

Compression devices reimbursement is reported by $20 \%$ of the responders and appears to be possible but with some limitations in $16 \%$. Italian responders reported no reimbursement in more than $80 \%$ of cases (Figure 13A, B).

Only the Bolzano local Health System reimburses compression bandages, zinc oxide bandages and compression stockings. Moreover, the reimbursement for a compressive bandage in Italy is only $8 € .^{16}$

It is curious how so an important social problem, involving many subjects $(0.1-0.3 \%$ of the general population, $1 \%$ at 60 years of age, till $5 \%$ at 90 years), prevalently belonging to the socially most deprived population, it is so poorly considered by Italian NHS, that spends a great amount of resources on venous related diseases ( $1 \%$ of the entire Health System budget) without a coherent program. ${ }^{17-19}$

\section{Question 14: What value of pressure $(\mathrm{mm} \mathrm{Hg})$ at the ankle is expected from your compression treatment? About:
a. 20
b. 30
c. 40
d. $50 \mathrm{~mm} \mathrm{Hg}$

The following standard has been suggested recently: mild ( $<20 \mathrm{mmHg}$ ), moderate $(\geq 20-40$ $\mathrm{mmHg}$ ), strong ( $\geq 40-60 \mathrm{mmHg}$ ), very strong ( $>60 \mathrm{mmHg}$ )..${ }^{20}$ Pressures $\geq 40 \mathrm{mmHg}$ are generally recommended for the treatment of venous leg ulcers. ${ }^{21}$

Our survey indicates that $40 \%$ of care providers use a strong level of pressure exerted by compression tools. Forty-seven percent of responders use a moderate pressure; only $14 \%$ of caregivers employ mild compressions, most likely those using knee stockings (Figure 14).

A sufficient compression pressure seems to be used by the majority according to some consensus and meta-analysis, ${ }^{1-10,16,20}$ however pressure value is mostly theoretical as is very 


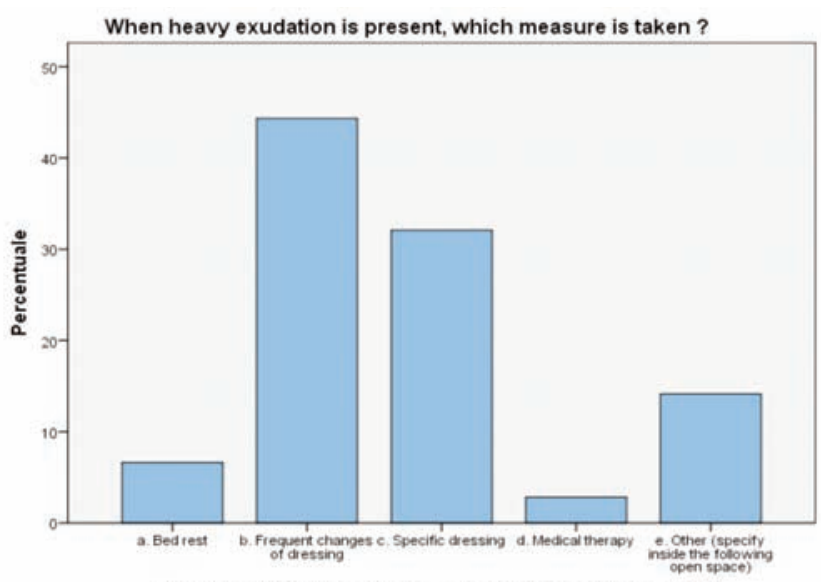

When heavy exudation is present, which measure is taken ?

Figure 7. Best therapeutic options in case of heavy exudation.

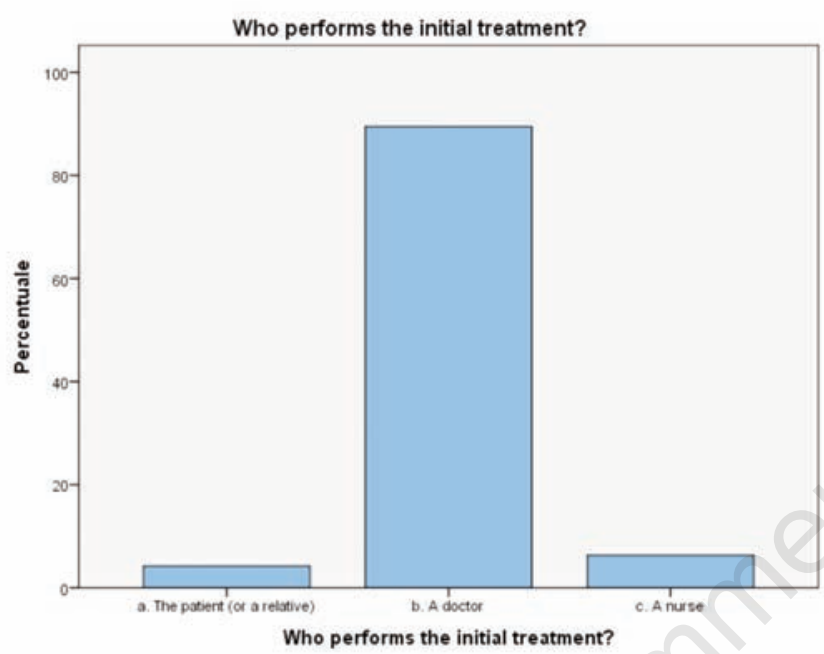

Figure 8. Who performed the initial treatment.

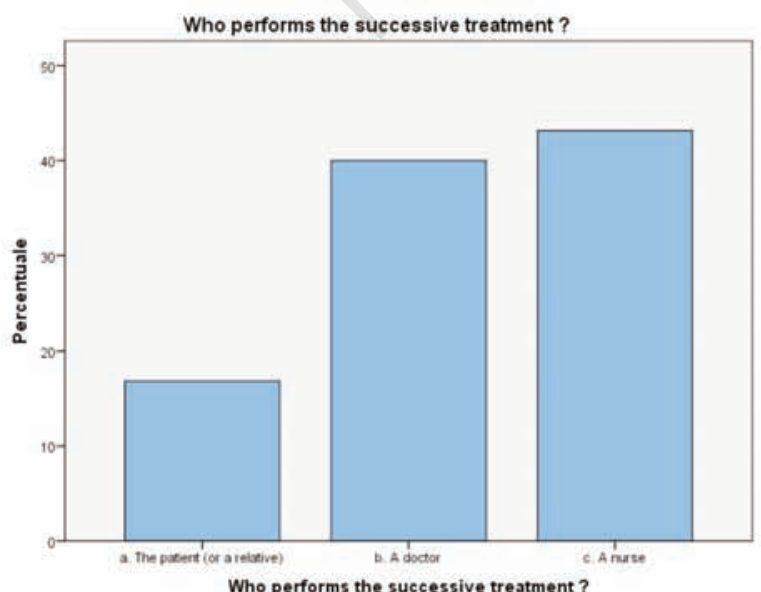

A

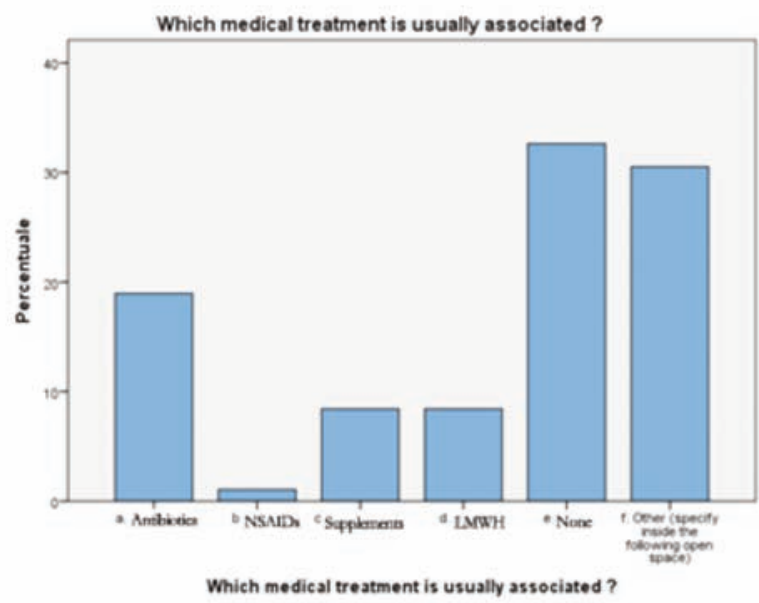

Figure 10. Associated medical treatments.

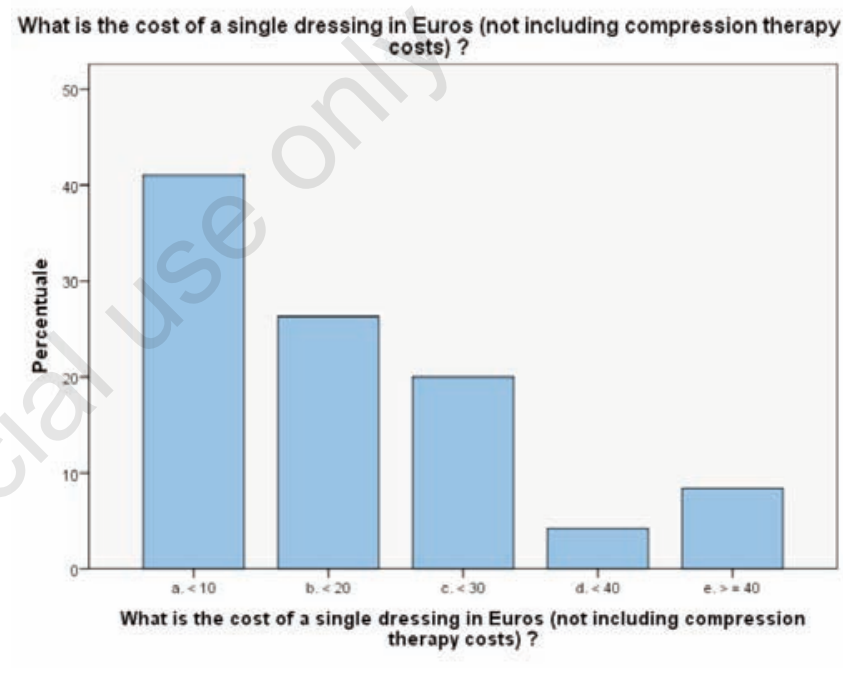

Figure 11. Cost of a single dressing (Euro), not including compression items.

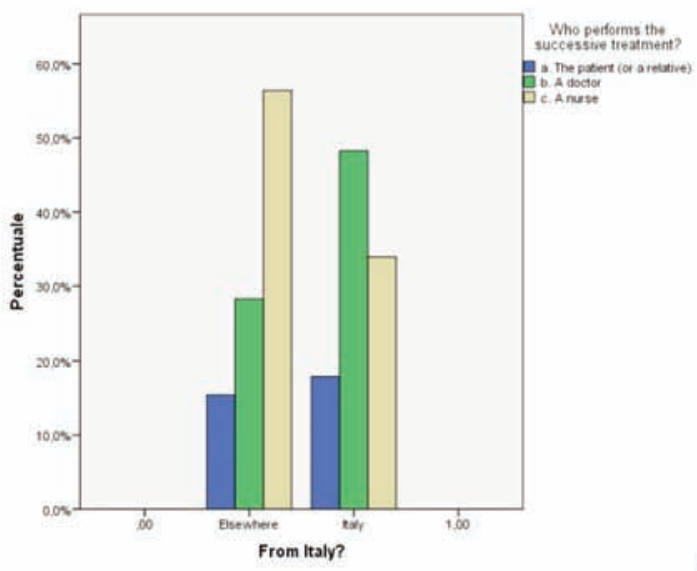

Figure 9. Who perform the successive treatment. 
Does the National Health Service of your country reimburse DRESSING charges,

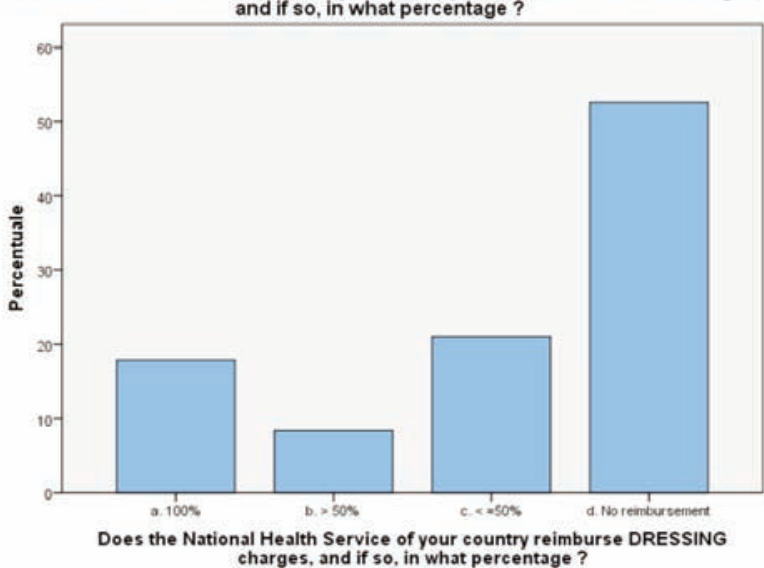

Figure 12. Percentage of reimbursements by different national health systems.

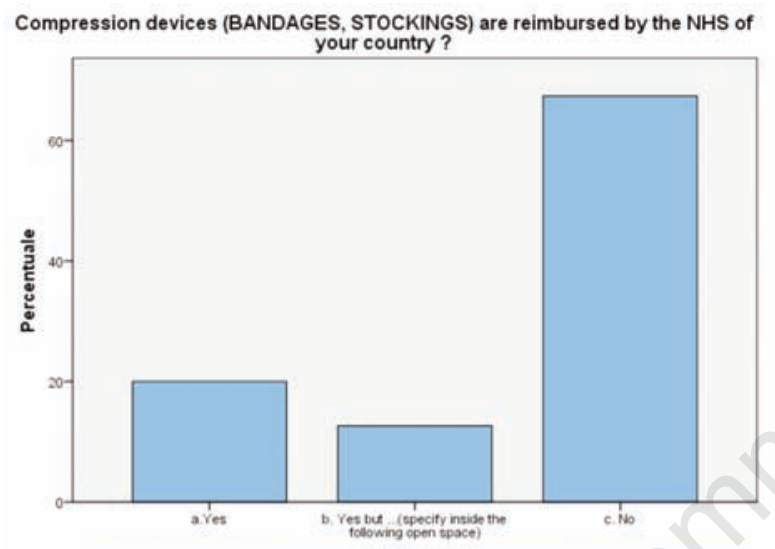

Compression devices (BANDAGES, STOCKINGS) are reimbursed by the NHS of your country?

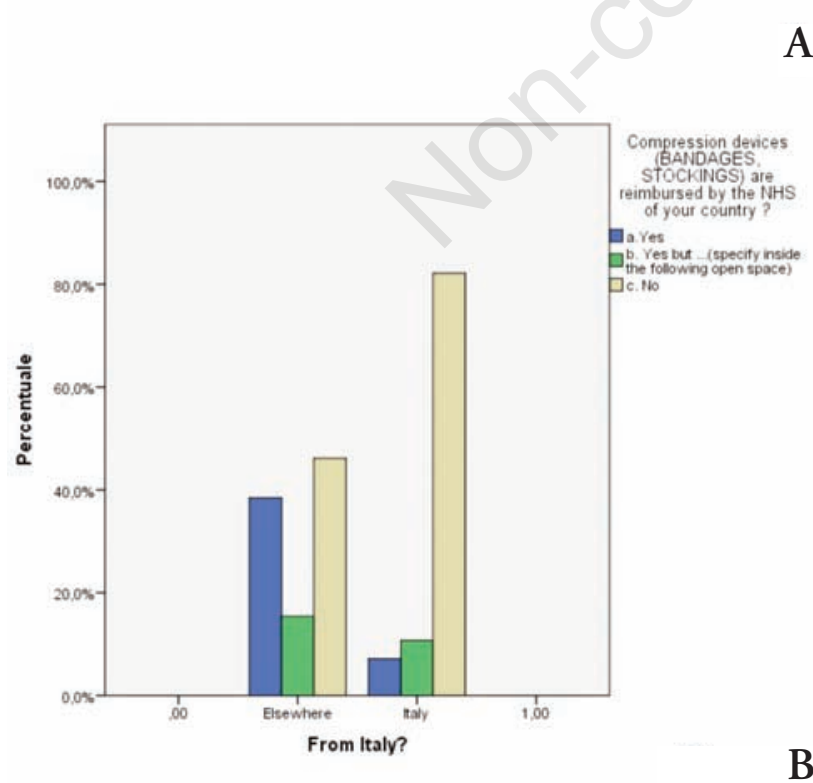

B

Figure 13. Percentage of reimbursements of compression devices (bandages, stockings) by different national health systems.

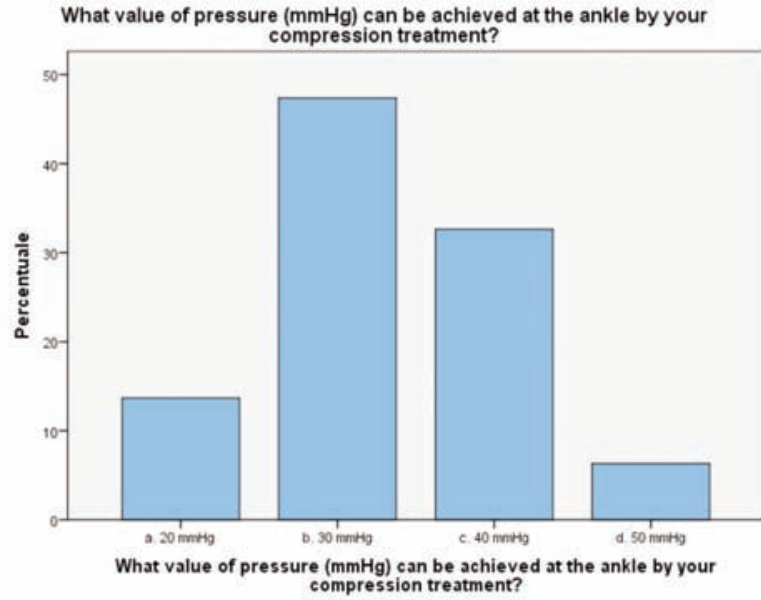

Figure 14. Expected pressure $(\mathrm{mm} \mathrm{Hg})$ at the ankle from the compression treatment different national health systems.

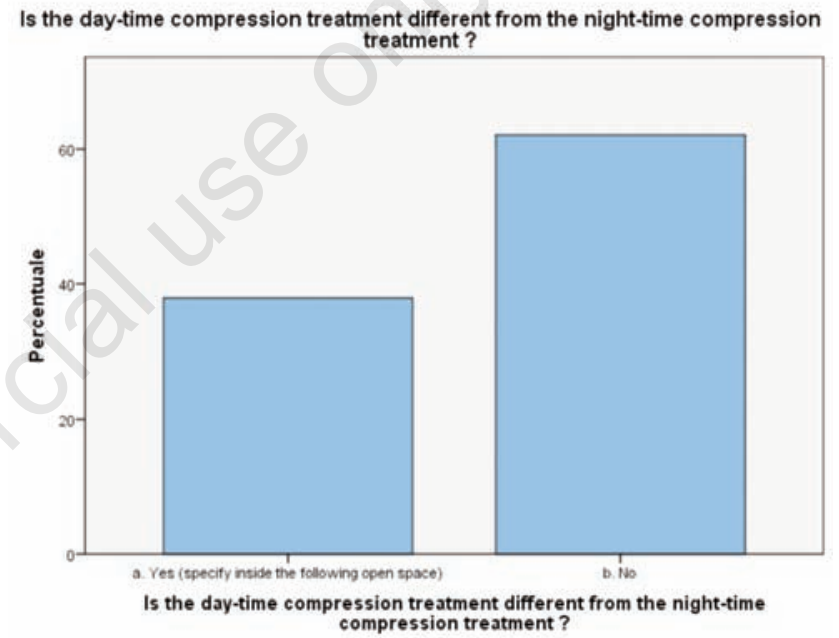

Figure 15. Differences between day-time and night-time compression treatments different national health systems.

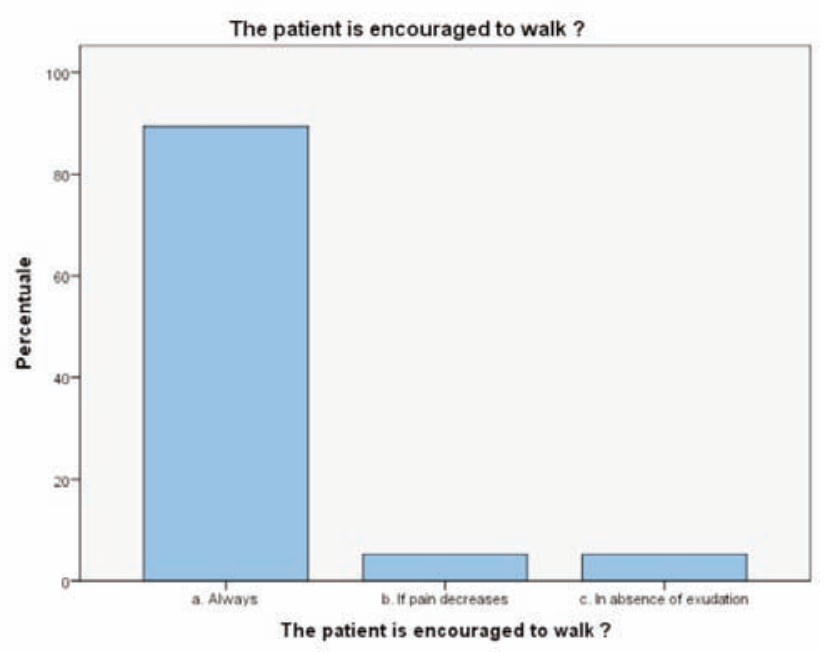

Figure 16. Cases in which the patient is invited to walk. 
rarely measured. ${ }^{4}$ Furthermore, while compression stockings have a pressure declared by manufacturing company (which correspond to an approximate value on the leg), the pressure of the bandages can be extremely variable only depending on the stretch applied to the bandage itself. $^{3}$

\section{Question 15: Is the day-time compression treatment different from the night-time compression treatment?}

a. Yes (specify inside the final open space) b. No

As shown in Figure 15, 62\% of respondents maintain the same compression day and night. Of those suggesting a lower compression at night, about half use a double layer ulcer kit, leaving only the inner layer at night (about 15$18 \mathrm{mmHg}$ ); the same for those using a multilayer bandaging. Two replies suggest the use of anti thrombus stockings at night.

If the same compression is used day and night without giving discomfort during resting it is presumably made by a short stretch bandages (that have a high working pressure, but a lower resting one) or, alternatively by a low pressure compression with limited effect at rest. ${ }^{20}$

Curiously, this problem has been rarely considered in consensus papers, till now.

\section{Question 16: Is the patient invited to walk?}
a. Always
b. If pain less
c. In absence of exudation
d. Never

As expected, $90 \%$ of survey responders always invite ulcer patients to walk. At the opposite, $10 \%$ of caregivers indicate some exceptions, finding that bed rest may be useful to reduce pain (5\%) and exudation (5\%) when they are particularly intense and can't be reduced by proper compression (Figure 16).

Patients with chronic venous insufficiency should walk - a truism that do not need to be discussed - (provided that an adequate compression is administered). Muscular venous pumps activation assisted by bandages or stockings, limits venous pooling and normalizes walking pressure, allowing the physiological healing mechanisms. Bed rest seems to be a very simple treatment modality for the initial stage of painful or heavily exudating ulcers, allowing reducing the frequency of dressing renewal and the assumption of pain killing medications. According to Bassi it was the oldest traditional method for treating ulcers when compression was not available..$^{22}$ Although this option doesn't fit the modern way of life and consequently it's rarely practiced, it is very efficient and may still be indicated in specific instances.
Question 17: When an ABI (Winsor Index) $<1$ is present, do you apply the same compression?

a. Yes

b. No (specify inside the final open space)

When an arterial impairment occurs (ABPI $<1) 74 \%$ of respondents do not change compression methods. Most of those (26\%) who change compression system suggest $\mathrm{ABPI}<0.5$ as cut-off for compression treatment, while few others chose a higher cut-off $(<0.7)$. Three participants suggest paying attention to absolute pressure $(80-90 \mathrm{mmHg}$ the limit) at the ankle instead of using ABPI; another respondent suggests considering post effort ABPI. Two suggest using only short stretch bandages. Some use a lower pressure of $20 \mathrm{mmHg}$ in case of arterial disease.

Most respondents do not follow traditional scholastic contraindication for compression treatment in limbs with arterial disease. According to a ' 92 Consensus Document, only critical limb ischemia (ankle systolic pressure $\leq 50 \mathrm{mmHg}$ and/or a toe systolic pressure of $\leq 30 \mathrm{mmHg}+$ necrotic lesions or recurrent rest pain) should contraindicate $a$ priori the compression therapy. ${ }^{23}$ Short stretch bandages applied without tension and providing moderate pressure, ${ }^{4}$ working only during ambulation could probably help even in extreme cases, by elimination of edema, allowing a better oxygen availability to tissues so enhancing arterialvenous gradient. ${ }^{24}$

\section{Question 18: Did you like this survey?}

The last question was: Did you like this survey? Over 105 forms received $75 \%$ replied yes; $16 \%$ absolutely yes; $9 \%$ no. Like always in these case, the most interesting aspect is the analysis of the reasons why some respondents did not like: 3 responders criticized that the etiology is not specified - but the indication venous ulcers was already present in the title; 4 thought that questions were too much generic - but this is not easily avoidable in this kind of surveys ( 2 without comment).

\section{Conclusions}

Organizing a survey, we tried to cover the most important aspects asking for average behaviors, and in a specific field (the venous ulcer), in a foreseen time of $5 \mathrm{~min}$.

The analysis of the respondents is not an indication to how to treat ulcers, but rather to how ulcers are treated in Italy, with a small international sample for comparison. Experience-based evidences (namely those provided by consensus conferences) give so variable indications on venous ulcers treatment that it is impossible to state which replies are adequate and which are questionable; however some contradictions have been pointed out commenting the respective survey answers. The only solid conclusion is the lack of interest of the Italian NHS to the ulcer problem revealed by the limited reimbursements offered for ulcer care, bandages and hosiery, that could be at the origin of an under treatment of the venous insufficiency and its consequences in our country.

The research main weakness is related to the small number of participants particularly of other countries that is related to the traditional difficulty of this kind of researches. Moreover, few more precise and specific questions could have been of some help (private activity, office versus hospital practice, pressure measurement, etc). This study could be useful as a base in case larger and more accurate surveys should be undertaken.

\section{References}

1. 0`Meara S, Tierney J, Cullum N, et al. Four layer bandage compared with short stretch bandage for venous leg ulcers: systematic review and meta-analysis of randomised controlled trials with data from individual patients. BMJ 2009;338:b1344.

2. World Union of Wound Healing Societies (WUWHS). Principles of best practice: Compression in venous leg ulcers. A consensus document. London: MEP Ltd; 2008.

3. Mosti G. Elastic stockings versus inelastic bandages for ulcer healing: a fair comparison? Phlebology 2012;27:1-4.

4. Management of chronic venous ulcers [database on the Internet]. Scottish Intercollegiate Guidelines Network; 2010. Available from: http://www.sign.ac.uk/pdf/ sign120.pdf

5. O'Meara S, Cullum NA, Nelson EA. Compression for venous leg ulcers. Cochrane Database Syst Rev 2009:CD 000265.

6. Robson MC, Cooper DM, Aslam R, et al. Guidelines for the treatment of venous ulcers. Wound Repair Regen 2006;14: 649-62.

7. Schultz GS, Sibbald RG, Falanga V, et al. Wound bed preparation: a systematic approach to wound management. Wound Repair Regen 2003;11 Suppl 1:S1-28.

8. Kahle B, Hermanns HJ, Gallenkemper G. Evidence-based treatment of chronic leg ulcers. Dtsch Arztebl Int 2011;108:231-7.

9. Palfreyman S, Nelson EA, Michaels JA. Dressings for venous leg ulcers: systematic review and meta-analysis. BMJ 2007; 335:244.

10. The Royal College of Nursing (RCN). Clinical Practice Guidelines. The nursing management of patients with venous leg 
ulcers. London: The Royal College of Nursing; 2006. Available from: http://www. rcn.org.uk/development/practice/clinicalguidelines/venous_leg_ulcers

11. Molan PC, Betts JA. Clinical usage of honey as a wound dressing: an update. $\mathrm{J}$ Wound Care 2004;13:353-6.

12. Franceschi C, Passariello F. Low cost medication for venous ulcer. Sugar-Honey: An online Vasculab Survey. Acta Phlebologica 2009;10:41-4.

13. Mancini S. Manuale di Flebologia. Colle Val d'Elsa: Laris Editrice; 2009.

14. Ertl P. How do you make your treatment decision? Prof Nurse 1992;7:543-52.

15. Ramelet A-A, Monti M. Phlebology: the guide. Amsterdam; New York: Elsevier; 1999.

16. Mosti G, Mattaliano V, Polignano R,
Masina M. La terapia compressiva nel trattamento delle ulcere cutanee. Linee Guida. Acta Vulnologica 2009;7:113-35. [Article in Italian].

17. Canonico S, Gallo C, Paolisso G, et al. Prevalence of varicose veins in an Italian elderly population. Angiology 1998;49: 129-35.

18. Callam MJ, Harper DR, Dale JJ, Ruckley CV. Chronic leg ulceration: socio-economic aspects. Scott Med J 1988;33:358-60.

19. Campitiello F, Lauriello C, eds. Percorsi diagnostico-terapeutici ospedale-territorio per la gestione delle ulcere cutanee. Torino: AIUC - Associazione Italiana Ulcere Cutanee; 2012 [In Italian]. Available from: http://www.aiuc.it/upload/ documenti/9/86/PERCORSI_DIAGNOSTICO-TERAPEUTICI.pdf
20. Partsch H, Clark M, Mosti G, et al. Classification of compression bandages: practical aspects. Dermatol Surg 2008;34: 600-9.

21. Martinez MJ, Bonfill X, Moreno RM, et al. Phlebotonics for venous insufficiency. Cochrane Database Syst Rev 2005:CD 003229.

22. Bassi G. Compendio di terapia flebologica. Torino: Editore MM; 1985.

23. Second European Consensus Document on chronic critical leg ischemia. Eur J Vasc Surg 1992;6 Suppl A:1-32.

24. Mosti G, Iabichella ML, Partsch H. Compression therapy in mixed ulcers increases venous output and arterial perfusion. J Vasc Surg 2012;55:122-8. 